\title{
The prebiotic effect of $\alpha-1,2$ branched, low molecular weight dextran in the batch and continuous faecal fermentation system
}

\begin{abstract}
The aim of this study was to establish the effect of smaller molecular weight $(0.5$ and 1.0 $\mathrm{kDa}$ ) on prebiotic efficacy and its putative sustainability in the human gut. The prebiotic effect of $\alpha-1,2$ branched, 0.5 and $1 \mathrm{kDa}$ dextrans were evaluated in faecal batch fermentations as compared with inulin. Both dextrans induce similar selectivity towards Bifidobacterium sp., Lactobacillus/Enterococcus and Bacteroides/Prevotella, and producing similar concentrations of short chain fatty acids. However, the $0.5 \mathrm{kDa}$ dextran was fermented faster than the $1 \mathrm{kDa}$ dextran, where both produced lower amount of gas than inulin. The fermentation of $1 \mathrm{kDa}$ dextran was further investigated in continuous gut models. The dextran increased Bifidobacterium and Roseburia sp. populations in the final vessel, while decreasing Clostridium histolyticum and Faecalibacterium prausnitzii. Overall, the $\alpha-1,2$ branched, 1 $\mathrm{kDa}$ dextran induced selective effect on the gut microbiota and stimulated short chain fatty acids, indicating prebiotic sustainability in distal regions of the gut.
\end{abstract}

Keyword: Prebiotics; Gluco-oligosaccharides; Faecal fermentation; Gut microbiota 